\title{
Comment on "Incidence and risk factors for osteoporotic fractures in patients with systemic lupus erythematosus versus matched controls"
}

\author{
Shih-Wei Lai ${ }^{1,2}$
}

${ }^{1}$ Department of Family Medicine, China Medical University College of Medicine, Taichung; ${ }^{2}$ Department of Family Medicine, China Medical University Hospital, Taichung, Taiwan

Received: July 16, 2019

Accepted: August 18, 2019

\section{Correspondence to}

Shih-Wei Lai, M.D.

Department of Family Medicine, China Medical University College of Medicine, No. 2, Yu-De Road, Taichung City, 404, Taiwan

Tel: +886-4-2205-2121

Fax: +886-4-2203-3986

E-mail:wei@mail.cmuh.org.tw https://orcid.org/0000-00027420-1572
Recently, a cohort study conducted by Kim et al. [1] published in The Korean Journal of Internal Medicine reported that systemic lupus erythematosus was associated with an increased risk of osteoporotic fractures when compared with non-lupus (hazard ratio, 2.9; 95\% confidence interval, 2.7 to 3.1). While Kim et al's study reported an increased relative risk of osteoporotic fractures (a relative risk of 2.9), the attributable risk was about 125 cases of osteoporotic fractures per 10,000 person-years of follow-up, or approximately one additional case of osteoporotic fractures per 80 persons per year. One cohort study reported that the incidence of deep vein thrombosis was 13.3-fold higher in the systemic lupus erythematosus group than the non-lupus group (15.1 vs. 1.1 per 10,000 person-years) [2]. The attributable risk was about 14 cases of deep vein thrombosis per 10,000 person-years of follow-up, or approximately one additional case of deep vein thrombosis per 714 persons per year. The relative risk was high in deep vein thrombosis versus in osteoporotic fractures (13.3 vs. 2.9), but the attributable risk was higher in osteoporotic fractures than in deep vein thrombosis (125 vs. 14 per 10,000 person-years). When making a decision in medical policy or practice, one should depend on the attributable risk rather than the relative risk in the aspect of the influence to public or individual health. Although the relative risk of deep vein thrombosis is higher, its attributable risk is lower than that of osteoporotic fractures. From the view of public health, physicians who participate in the care of patients with systemic lupus erythematosus should make their first priority to consider the risk for osteoporotic fractures. We agree with Kim et al's comments that strategies to prevent osteoporotic fractures in patients with systemic lupus erythematosus are a future research direction.

\section{Conflict of interest}

No potential conflict of interest relevant to this article was reported.

\section{REFERENCES}

1. Kim CS, Han KD, Jung JH, et al. Incidence and risk factors for osteoporotic fractures in patients with systemic lupus erythematosus versus matched controls. Korean J Intern Med 2021;36:154163.

2. Chung WS, Lin CL, Chang SN, Lu CC, Kao CH. Systemic lupus erythematosus increases the risks of deep vein thrombosis and pulmonary embolism: a nationwide cohort study. J Thromb Haemost 2014;12:452-458. 